\title{
Design of intelligent logistics information platform based on wireless sensor RFID technology
}

\author{
Hai $Y e^{1, a}$ \\ ${ }^{1}$ Qingyuan Polytechnic, Guangdong Qingyuan, 511510, China \\ ayehaiqyzyjs@163.com
}

Keywords: Intelligent logistics; RFID; Wireless sensor network; Information platform; Electronic product code

\begin{abstract}
The core technology of RFID is the label of EPC (electronic product code), because EPC provides unique identification of physical objects, the use of EPC can achieve the tracking and monitoring of goods in intelligent logistics information. This paper deeply analyzes the wireless sensor network and RFID technology in the application of intelligent logistics system. The paper presents design of intelligent logistics information platform based on wireless sensor RFID technology. The experimental results show that this technology can improve efficiency of logistics system effectively.
\end{abstract}

\section{Introduction}

RFID technology will solve the problem of automation of information acquisition in logistics management. Attached to the single commodity, carton or pallet on the RFID label, can provide two-way communication product flow and information flow in supply chain management, and through the Internet transmission of data collected from the label. Compared with the bar code technology, RFID technology can greatly reduce the labor cost to obtain product information, so that the automation of many aspects of the operation of supply chain.

For retailer's inventory and cost of sales accounted for their operating costs of $2 \% \sim 4 \%$. Using the reader to read the pallets, containers, and it is boxes and individual items, so as to replace the bar code identification of human consumption [1]. RFID technology can make the sales staff numbers reduced by more than $30 \%$. Reduce the code reading labor costs using the product grade RFID can help retailers to reduce labor costs as well as regular cargo management and shelf storage products service fee. For RFID products, by improving the self - service, reduce the inspection time and error, can improve the current this kind of automatic scan checks.

Wireless sensor network is established according to the relationship between routing and data transmission time can be divided into proactive routing protocol, on-demand routing protocol and hybrid routing protocol. Active routing establishment and maintenance of the routing overhead, requiring high computational resources; routing, on-demand routing in transmission before delay; hybrid routing is the comprehensive utilization of these two ways. According to whether the node addressing and it is whether to address identifies the destination, can be divided into routing protocol address and address non routing protocol based on it [2]. Address based routing is common in traditional routing protocols, while WSNs is generally not used alone and combined use with other strategies.

In the warehouse, the most widely used RF technology is the access of goods and inventory; it can be used to realize the automation of inventory and pickup operation. In the warehouse management, the supply chain planning system for receiving plan, plan, plan and other shipment pick-up combined with RFID technology, can efficiently complete a variety of business operations, such as the designated storage area, shelves pickup and replenishment etc.. In this way, enhances the operation accuracy and speed, improve service quality, reduces the cost, saves labor and inventory space, while reducing the 
commodity mislaid, sent the wrong, theft, damage and inventory and delivery error caused by the loss due to the whole logistics.

The antenna height from the ground and closer, the loss factor is bigger, this is unfavorable factors to design sensor network physical layer. However, some inherent characteristic of wireless sensor network is also conducive to the design of wireless sensor network, for example, high-density deployment with diversity characteristics, can be used to overcome the shadowing and path loss. The paper presents design of intelligent logistics information platform based on wireless sensor RFID technology.

\section{Development and design of intelligent logistics information management platform by RFID}

The core technology of RFID is the label of EPC (electronic product code), because EPC provides unique identification of physical objects, so the use of EPC can achieve the goods in the whole cold chain logistics tracking, monitoring and RFID temperature tag can also provide temperature, ensure the quality and safety of goods RFID technology in cold chain logistics application: fresh food cold chain logistics. Application of RFID technology, the fresh food became cheaper shopping guide network from production; it is in the supply chain of the whole flow process will be timely, accurate tracking, transparency.

In the full understanding of inventory management business needs, combined with RFID technology, transformation and the existing business process redesign [3]. Business processing mode is similar to the original model, but the mode of operation on a handheld device for. System operation into each key link of operation, so that the operator can interact with the system to obtain the real-time information system, support system can real-time acquisition to key operation data for correlation system for fast and effective treatment.

RFID tag stores a unique code to mark the reality of the object, resulting in the business process related information data stored in a container, when required in the code as the query value to obtain relevant information through network [4]. RFID technology can also identify multiple tags, a quick recognition speed, combined with Internet technology, can realize the information sharing in the global range, as is shown by equation (1).

$$
\bar{w}(m, s):=\sum_{j=1}^{s-1}\left(\prod_{i=s-1}^{j} A(m M+i)\right) w(m M+j-1)+w(m M+s-1)
$$

In the receiving area using a RFID encoder (if needed) and receiving operation, require stacked in all cargo receiving areas must have a single tray, tray number with barcode label way paste on the tray if SO has not yet been served, the system indicates the goods into the buffer.

Warehousing logistics current, most of the use of bar code as intelligent storage management style, although its intelligent degree is greatly improved than before, but still need to spend a lot of manpower and material resources into warehousing logistics. Now if the RFID system with the existing bar code system are combined, which can effectively solve the problem with the warehouse and cargo flow information related to the management, not only can increase the number of processing goods within one day, all the flow of information can also see these goods. The bar code technology combined with the RFID is in a way of the current application [5]. It can be affixed to the goods barcode, radio frequency electronic label on the goods storage tray or forklift, electronic tag storing all the items of information on the tray or forklift, as is shown by equation (2).

$$
\mu_{m}(k)=\beta(k) s_{0}(k)+\alpha(k)
$$

With the gradual popularization and application of maturity and system of Internet technology popularization, more and more applications will involve equipment category is more and more wide, 
which relates to the acquisition device (such as a RFID reader, all kinds of sensors) variety, how to maximize the reduction of application software development cycle, the unified management of different hardware equipment.

The application of RFID in the fresh food inventory management can reduce the artificial audit work, but it can ensure the safety of the storage of goods quality, reduce the cost of management. For the retailer RFID Technology: fresh food cold chain logistics application, when the automatic replenishment system show the need for replenishment, you can order to immediately upstream enterprises, through the feasible solution of RFID and RFID technology to ensure the required cargo security, arrive on time, so as not to appear short or shortage.

The purchasing department is to the receiving application form, and prints the material receiving application form of bar code, and then transported to the warehouse material [6]. Warehouse personnel to use the wireless mobile device (handheld) scanning receiving application form of bar code, based on the wireless mobile device receiving application form the scan from the warehouse management system to collect material information application download single material, material information including: material bar code, material name and quantity of goods received, and specify the material whether is urgent and whether you need to test.

In this paper, the RFID technology combining with the Internet of things, the use of RFID electronic tags as product identification means, with information network to obtain product storage material use and automatically generate a single storage, according to the traditional logistics warehousing management exists in the product identification two bottlenecks and product information acquisition, the structure and function of the system of the analysis.

\section{Wireless sensor RFID technology}

This management platform is used to monitor the use of energy in wireless sensor networks and mobile node and task management. They can help the premise of sensor nodes in low energy consumption under certain monitoring task collaboration. Energy management platform can manage a node how to use its energy.

WSN in the plane structure, grade and level, there is not any difference; bottleneck problem does not exist in principle, have good robustness. The disadvantage is the scalability, maintenance of dynamic change of the need to control a large number of routing information.

The sensor module is responsible for the information in the monitoring area acquisition and data conversion, sensor type is determined by the physical signal monitored form, such as for platinum resistance temperature sensor for monitoring the pressure sensor, the capacitive sensor; processor module is responsible for the control of the whole sensor node operation, storage and processing of the collected data and itself send data to other nodes, as is shown in equation (3) [7].

$$
\nabla^{2} u=\frac{\partial^{2} u}{\partial x_{1}^{2}}+\frac{\partial^{2} u}{\partial x_{2}^{2}}
$$

When mobile beacon moves in the location area, and it is moving to a plurality of the new position, immediately in the position of a supplement to a common node. According to the real-time location information of beacon nodes, ordinary new position knows their position [8]. They are equivalent to the beacon. In addition, increasing the common node at the same time, but also improve the connectivity of random network. The introduction of the mobile beacon advantage is even only one beacon can also realize the localization of unknown node.

RFID module design of passive mode, one of the most basic requirements requires low power consumption characteristics, in order to improve the life of the card and expand applications, can be said to reduce power consumption, with the guarantee of effective communication distance is as important. Therefore, the chip card generally use very demanding low power technology and high 
efficient energy saving technology, such as circuit design is adopted in "sleep mode" design technology.

\section{Design of intelligent logistics information platform based on wireless sensor RFID technology}

Used to do not overlap each other in space area placed inventory items, warehouse, usually a library position can display multiple inventory; can also be a larger inventory occupied several storage. Warehouse equipment: for warehouse management equipment, such as forklifts, trolleys; in a large and busy warehouse needs to carry on the reasonable dispatch, real-time positioning of these devices, to improve equipment utilization rate.

RFID technology is based on modern science and technology, to identify, collect, record, distribution, management of goods by using radio frequency identification technology, used to the RFID technology can be more accurate, simpler, more convenient and more advanced modern logistics management mainly exist in each link, the production, distribution, retail enterprises [9].

With the aid of the RFID technology, the company also can realize the raw materials, semi-finished products, finished products, transportation, warehousing, distribution, shelves and final sales, real-time monitoring and even return processing chain, so the reasonable control of inventory, to realize intelligent management of logistics, as is shown by figure1. Wireless sensor networks are often applied to logistics management information on the high real-time requirement of the field, timely detection, processing and transmission of information is the essential requirement. To provide real-time guarantees MAC layer and other layers of cooperation.

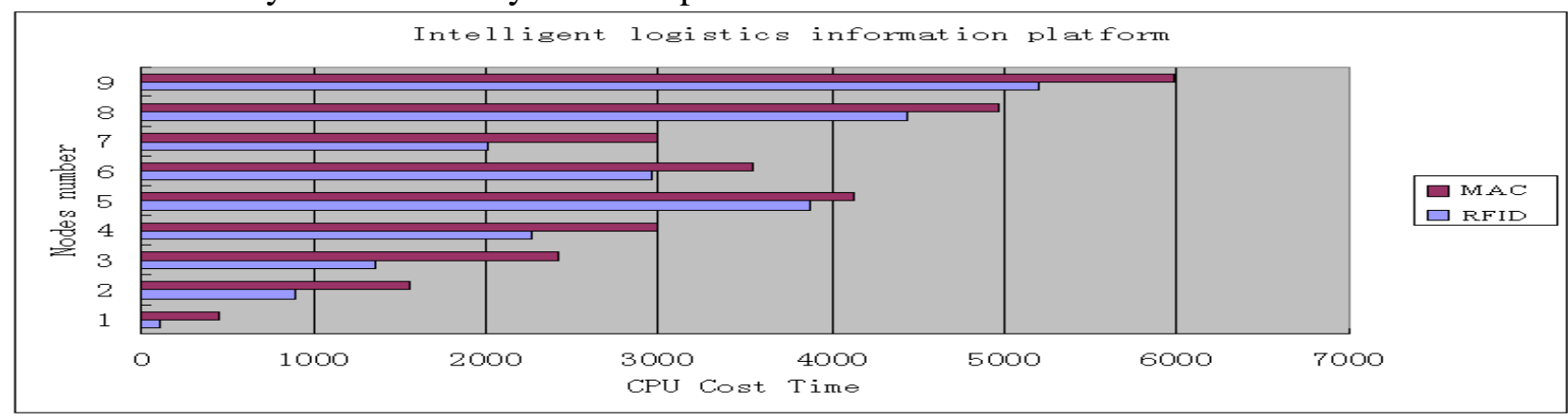

Figure. 1 Design of intelligent logistics information platform based on RFID technology with MAC

In this paper, the experimental RFID technology of Internet of things based on the optimization and improve the application of each link in the supply chain management, including the raw material supply, production, fixed asset management, inventory management, sales, after-sales service and so on many aspects, the research of RFID technology for the optimization and improvement of supply chain structure, improve the management performance of supply chain and improve the transparency of the supply chain in the role of. According to the characteristics of modern logistics, put forward a system framework of intelligent logistics distribution based on the Internet of things, can improve the efficiency of logistics system effectively.

\section{Summary}

At present no inter node measurements of absolute distance or range, thus reducing the RFID requirement of wireless sensor networks for node hardware, making the node cost is more suitable for large-scale sensor networks. The paper presents design of intelligent logistics information platform based on wireless sensor RFID technology. RFID technology is economical and practical, but the disadvantage of RFID is also very prominent: cost and investment is too high. Although the label costs continued to decline in recent years, but the label and the identifier of the cost is still relatively high, this has become the main obstacle to wider application of RFID. 


\section{References}

[1] SunYu Jie, Julian Clive, "Research of Logistics Product Intelligent System Distribution Based on Internet of Things", JDCTA, Vol. 7, No. 6, pp. $979 \sim 986,2013$

[2] Seung-Jung Shin, Jung-Hwa Kang, and Kae-Dal Kwack, , "A Study of a Protocol Model for Information Security of the RFID Base Individual Identification Device", RNIS, Volume 7, pp. 10 12,2011

[3] Lvqing Yang, Caili Wang, Wenhua Zeng, "Research of Matrix-based Grouping Method on Anti-collision Algorithm for RFID Tag Identification", AISS, Vol. 5, No. 13, pp. 126 133, 2013.

[4] Lu Wang, Wusong Wen, "Path Planning for Mobile Beacon Nodes in Localization for Wireless Sensor Networks", JDCTA, Vol. 7, No. 3, pp. 16 24, 2013.

[5] Wenbo Zhang, Yongxin Feng, "Energy Efficient Topology Control Conserving Algorithm for Wireless Sensor Networks", IJACT, Vol. 5, No. 5, pp. $142 \sim$ 149, 2013.

[6] Liqun Shan, , Jinkuan Wang, Ruiyan Du, Xiaojun Chu, "Multipath Data Aggregation Routing In Wireless Sensor Networks", AISS, Vol. 4, No. 8, pp. 344 352, 2012.

[7] Guo Wang, Dong Dai, "The Novel Model of Configuration and Performance Management System in Internet of Things by RFID and SNMP", JCIT, Vol. 8, No. 4, pp. $618 \sim$ 626, 2013.

[8] Wei Lifeng, Ji Jianwei , Xu Jiawang, "Research on the Production Logistics Information Terminal Based on RFID Technology", JDCTA, Vol. 6, No. 20, pp. 114 121, 2012.

[9] Jung Tae Kim, "Analyses of Integrated Security Framework with Embedded RFID System for Wireless Network Architecture", JCIT, Vol. 8, No. 14, pp. 188 194, 2013. 